\title{
AUTOMATIC GENERATION OF DYNAMIC MACRO-MODELS USING QUASI- STATIC SIMULATIONS IN COMBINATION WITH MODAL ANALYSIS
}

\author{
Lynn D. Gabbay and Stephen D. Senturia \\ Department of Electrical Engineering and Computer Science, Massachusetts Institute of Technology \\ Cambridge, MA 02139
}

\begin{abstract}
Reduced-order macromodeling can be used to simplify the simulation problem in microelectromechanical systems. In this paper, we report the successful implementation of a methodology for automatically generating reduced-order macro-models for the conservative energy-domain behavior of electrostatically actuated MEMS devices from 3D simulations. These models are directly usable in circuit- and system-level simulators for complete MEMS system design. This method has been successfully applied to the electrostatic actuation of a suspended beam and an elastically supported plate with an eccentric electrode and unequal springs (producing tilting when actuated).
\end{abstract}

Keywords: Macromodel, Modal analysis

\section{INTRODUCTION}

While several CAD systems have begun to enable quasistatic simulation for MEMS devices, it has proven difficult to implement dynamics simulation [1-2]. Microelectromechanical structures obey a complex set of equations of motion that must account for the tight coupling of the multiple energy domains of the system. The determination of dynamic behavior via full threedimensional simulation is computationally expensive. Furthermore, numerical dynamic simulation on fully meshed structures is too cumbersome to serve as the device model that can be part of a larger system of the designer's choosing, such as a black-box element controlled by a feedback circuit.

In this paper, we present a means by which a threedimensional model of a two conductor electromechanical device without dissipation can be converted into a reduced-order macromodel that can readily be inserted as a black-box circuit element into an analog circuit simulator. This process is based upon the energy method approach [3], in that we shall construct analytical models for each of the conservative energy domains of the system and determine all forces as gradients of the energy. The energy method approach has the advantage of making this process modular, enabling us to incorporate other energy domains into our

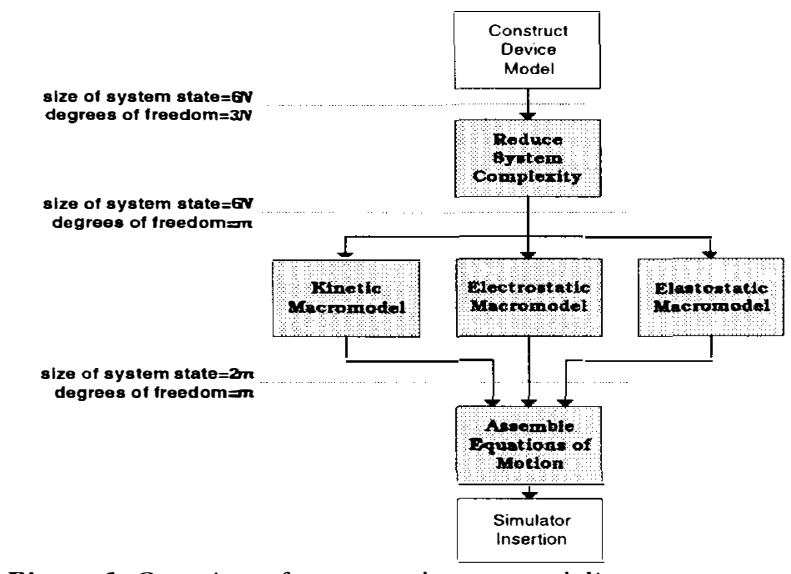

Figure 1. Overview of automated macromodeling process models in the future. Another benefit of energy methods is that the models we shall construct are guaranteed to be energy conserving, because the stored energies in each domain shall each be constructed as an analytical function, and all forces shall be computed directly from analytically computed gradients. This process also has the advantage of being able to be performed almost entirely automatically, requiring the designer only to construct the model, run a few full three-dimensional numerical computations, and set a few preferences a priori. Above all, this process has the ultimate benefit of constructing models that are computationally efficient, allowing their use in a dynamical system-level simulator.

\section{THEORY}

A high level description of our approach is depicted in Figure 1. Our first task is to reduce the degrees of freedom of the system. Rather than allow each node in a finite element model to be free to move in any direction, we constrain the motion of the system to a linear superposition of a select set of deformation shapes. This set will act as our basis set of motion. The positional state of the system will hence be reduced to a set of generalized coordinates, each coordinate being the scaling factor by which its corresponding basis shape will contribute. Next, we must construct analytical macromodels of each of the energy domains of the system. In the case of conservative capacitive electromechanical systems, these consist of the electrostatic, elastostatic, and kinetic energy domains. These macromodels will be analytical functions of the generalized coordinates. We can then use Lagrangian mechanics in order to construct the equations of motion of the system in terms of its generalized coordinates. Finally, we can translate these equations of motion into an analog hardware description language, thereby constructing a black-box model of the electromechanical system that can be inserted into an analog circuit simulator.

\section{MODEL COMPLEXITY REDUCTION}

The MEMS device is first constructed as a finite element model with $N$ free nodes. Neglecting node rotations, this system has $3 N$ degrees of freedom. The dynamical state of the system requires $6 N$ terms, where $3 N$ terms record the node positions, and an additional $3 N$ terms record the node velocities. For $6 N$ state terms, $6 \mathrm{~N}$ first order differential equations are needed to represent the equations of motion of the system. If $N$ is large, it is computationally expensive to integrate the equations in time.

To solve this problem, we restrict the motion of the system. Let us define $\psi$ to be a $3 N$ element vector representing the positional state of the system. We constrain the degrees of freedom of the system by declaring that $\psi$ is a linear superposition of $m$ linearly independent basis shapes $\varphi_{i}$ offset from an equilibrium state $\psi_{\text {eqm }}$. Note that this equilibrium state is the shape of the structure after any initial stress relaxation take place; naturally, if there are no initial stresses or the structure is constrained so as to prevent stress relaxation, the basic shape is already at its 
equilibrium position. Thus, we rewrite our representation of the state of the system as

$$
\psi=\psi_{\text {eqm }}+\sum_{i=1}^{m} q_{i} \varphi_{i}
$$

where $q_{i}$ are the coefficients of the basis shapes of the linear superposition. Henceforth, we refer to the $q_{i}$ as the generalized coordinates of the system. In effect, this constrains the description of the system from $3 N$ to $m$ spatial degrees of freedom. Correspondingly, this reduces the number of terms needed to represent the dynamical state of the system, and thus the number of first order differential equations in the equations of motion, to $2 \mathrm{~m}$. When $m \ll N$, this constitutes a significant computational advantage over the full $6 \mathrm{~N}$ representation.

When choosing the basis shapes, the designer is faced with two questions. The first is how to find candidate basis shapes. Because any complete basis set of a $3 N$ element vector can have $3 \mathrm{~N}$ linearly independent basis shapes, the second question is which shapes from the complete set should be used. There are numerous sources to construct the shapes among which we will choose. Ananthasuresh [4] demonstrated the use of mechanical harmonic mode shapes for use with MEMS device complexity reduction. Hung [5] presented a technique for using actual system motion to construct a set of representative basis shapes. Any or all of these methods are valid.

For this research, we use mechanical harmonic mode shapes for our basis shapes. Numerical modal analysis yields the eigenvalues $\varphi_{i}$ of the mechanical system, being the $N$ element vectors that describe the shapes of the modes of vibration, as well as the associated generalized frequencies $\omega_{i}$ and masses $m_{i}$. There are several advantages to choosing mode shapes for the basis set. For one, Ananthasuresh demonstrated that only a few mechanical mode shapes are necessary to accurately capture the motion of simple MEMS devices. Also, the mode shapes constitute a linearly independent set of basis shapes; thus it is not necessary to perform a back orthogonalization to confirm the independence of each additional mode shape. Furthermore, the modal formulation readily provides a representation for the kinetic energy of the system $T(\dot{q})$, given by

$$
T(\dot{q})=\sum_{i} \frac{1}{2} m_{i} \dot{q}_{i}^{2}
$$

and a linear representation for the elastostatic strain energy of the system $U_{\text {linear strain }}(q)$, given by

$$
U_{\text {linear strain }}(q)=\sum_{i} \frac{1}{2} m_{i} \omega_{i}^{2} q_{i}^{2}
$$

Once a set of $M$ basis shapes is chosen, we must choose the minimum number $m$ of those shapes necessary to characterize typical motion behavior of the structure. Our approach begins with a single coupled-energy-domain three-dimensional quasistatic simulation for the system under a typical example of actuation. Let us define $\psi_{\mathrm{ex}}$ to be the positional state calculated by a single quasistatic simulation. It is possible to determine the coefficients $c_{i}$ such that

$$
\psi_{\mathrm{ex}}=\psi_{\mathrm{eqm}}+\sum_{i=1}^{M} c_{i} \varphi_{i}
$$

If we assume that $\psi_{\mathrm{ex}}$ is an example of typical motion, then by sorting the shapes in decreasing order of contribution toward our example motion, we construct a prioritized list of which basis shapes will have the greatest significance on general overall motion. The designer can now decide which and how many shapes to use in the reduced model of the system. Furthermore, the magnitudes of the coefficients $c_{i}$ can be used to understand the relative expected magnitudes of the generalized coordinates during dynamics simulation. By providing $c_{i}$, we enable the designers to make an educated selection for the system operating range.

\section{MACROMODEL CONSTRUCTION}

The next step is to construct macromodels that replace the full 3D simulation for each of the system's energy domains. As mentioned above, the use of mode shapes automatically creates macromodels for the kinetic energy and linearized elastostatic energy domains. Here, we shall present how we construct macromodels for any function that would normally require full 3D simulation and then apply this technique to create macromodels for the electrostatic energy domain.

The requirements for a macromodel are that it must be an analytical function of the generalized coordinates and compare accurately to full three-dimensional simulation. The process by which we create such a macromodel is depicted in Figure 2. Full 3D simulation in the energy domain of interest is run several times for values of the generalized coordinates that adequately span the predetermined operating range for the system. Then, we select a generalized functional form, on which we then use the LevenbergMarquardt non-linear function fitting scheme [6] in order to determine the parameters that fit this generalized form to the appropriate stored energy function. This algorithm remains the same, regardless of the nature of the 3D simulation.

In order to construct the electrostatic macromodel, recall that electrostatic forces are produced by the charges that accumulate on the conductor surfaces of the MEMS device under an applied voltage. The force $\mathbf{F}_{e}$ that stores energy into the electrostatic domain of the system is given by the gradient of the electrostatic energy $U_{e}$ :

$$
\mathbf{F}_{e}=\nabla U_{e}
$$

The electrostatic energy $U_{e}$ and co-energy $U_{e}^{*}$ are given by

$$
U_{e}=\frac{1}{2} \frac{Q^{2}}{C} \quad U_{e}^{*}=\frac{1}{2} C V^{2}
$$

where $Q$ is the charge on the conductors, $C$ is the capacitance between the conductors, and $V$ is the applied voltage. It is generally advantageous for us to consider the co-energy, as we prefer to work in terms of voltage rather than charge. In this case, the gradient of the electrostatic co-energy $U_{e}^{*}$ is the force that draws energy out of the electrostatic domain. Thus, the force $\mathbf{F}_{e}$

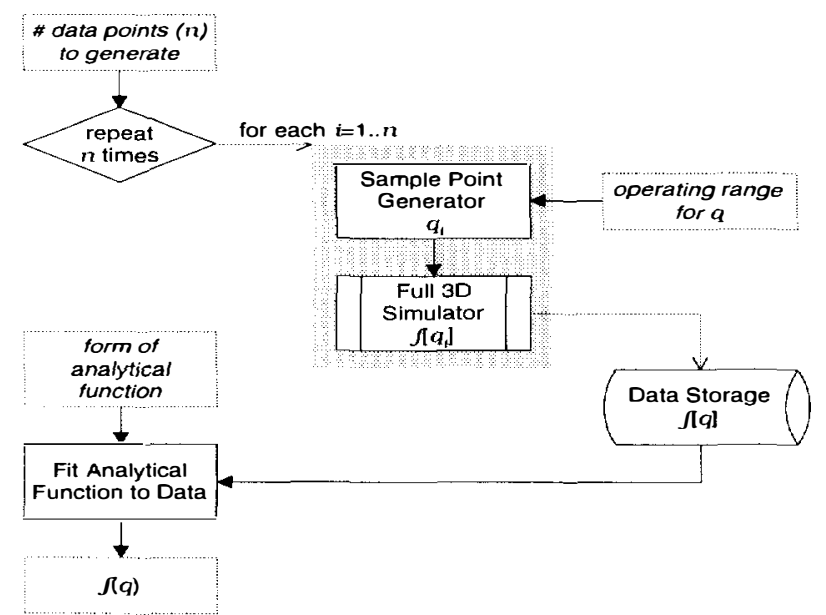

Figure 2. Macromodeling algorithm for full 3D simulation domain 
that puts energy into the electrostatic domain is given by

$$
\mathbf{F}_{e}=-\nabla U_{e}^{*}
$$

When comparing Equations 3 and 4, we note the sign change. Because we choose to determine our electrostatic forces from the electrostatic co-energy rather than energy, a sign change must be taken into account.

Let us return to determining the electrostatic forces. Because the applied voltage is independent of motion, the gradient need only be applied to the capacitance, thus:

$$
\mathbf{F}_{e}=-\left(\frac{1}{2} V^{2}\right) \nabla C
$$

In order to construct a macromodel of the electrostatic domain, we must construct an analytical model of the capacitance of the system. We shall use an arbitrary multivariate form to represent the capacitance, but it should be designed such that it can capture the non-linearities that can be expected from a capacitance function. Recall that the capacitance of a large parallel-plate capacitor neglecting fringe field effects is given by $\varepsilon_{0} A / d$, where $A$ is the area of the plate, and $d$ is the distance between the plates. Our generalized coordinates would most correspond to the gap $d$. Thus, it makes sense that our analytical form should have denominator terms. In this research, we use the form of a rational fraction of multivariate Taylor polynomials to represent the capacitance function. This form is given by:

$$
\frac{\sum_{i_{1}=0}^{R_{1}} \sum_{i_{2}=0}^{R_{2}} \cdots \sum_{i_{m}=0}^{R_{m}} a_{i_{1} i_{2} \cdots i_{m}} q_{1}^{i_{1}} q_{2}^{i_{2}} \cdots q_{m}^{i_{m}}}{\sum_{i_{1}=0}^{S_{1}} \sum_{i_{2}=0}^{S_{2}} \cdots \sum_{i_{m}=0}^{S_{m}} b_{i_{1} i_{2} \cdots i_{m}} q_{1}^{i_{1}} q_{2}^{i_{2}} \cdots q_{m}^{i_{m}}}
$$

Henceforth, we shall refer to this as a $\left[\begin{array}{lllllll}R_{1} & R_{2} & \cdots & R_{m} / S_{1} & S_{2} & \cdots & S_{m}\end{array}\right]$ model.

\section{ASSEMBLING THE EQUATIONS OF MOTION}

Given representations for the kinetic and potential energy domains of a system, we can use Lagrangian mechanics to construct the equations of motion [7]. Recall that the Lagrangian $L(q, \dot{q}, t)$ is a function of the general coordinates $q$, their first time derivatives $\dot{q}$, and time $t . L(q, \dot{q}, t)$ is defined by

$$
L(q, \dot{q}, t)=T(q, \dot{q}, t)-U(q, \dot{q}, t)
$$

where $T(q, \dot{q}, t)$ is the kinetic energy and $U(q, \dot{q}, t)$ is the potential energy of the system. The equations of motion come directly from Lagrange's equations, given by

$$
\frac{d}{d t}\left(\frac{\partial L}{\partial \dot{q}_{i}}\right)-\frac{\partial L}{\partial q_{i}}=0
$$

representing a set of $m$ equations, one for each generalized coordinate $q_{i}$. By incorporating the macromodels for the kinetic, elastostatic, and electrostatic energy domains, given by Equations 1,2 , and 5 respectively, and correctly accounting for the use of electrostatic co-energy instead of energy, our equations of motion become:

$$
m_{i} \ddot{q}_{i}=+\frac{1}{2} V(t)^{2} \frac{\partial C(q)}{\partial q_{i}}-m_{i} \omega_{i}^{2} q_{i}
$$

Because our representation of the capacitance is an analytical function, we can compute the gradients of these functions analytically rather than numerically. This averts the possibility of numerical error creating hidden energy sources or sinks, thereby creating or destroying energy arbitrarily within our equations of motion.
Finally, the resulting equations of motion are written to an analog hardware description language input file for the SABER simulation suite [8]. Note that all data extraction, macromodel generation, equation of motion assembly, and input file exportation are done automatically by computer. The initial investment of time to generate the circuit simulator input file need only be made once. The input file may then be used repeatedly for any number of dynamics simulations.

\section{RESULTS}

Here, we present the results of this process for the case of a simple fixed-fixed beam suspended above a fixed electrode strip. Both the beam and the electrode are assumed to be unstressed polysilicon conductors with a Young's modulus of $165 \mathrm{GPa}$ and a Poisson ratio of 0.23 , have dimensions of $100 \times 20 \times 0.5 \mu \mathrm{m}$, and are separated by a $2 \mu \mathrm{m}$ gap. Modal analysis is then performed to determine the first three modes. For our single quasistatic solution, we apply a voltage of 80 volts. By projecting the resulting actuated motion onto the mode shapes, we note that the first mode dominates the overall motion, with a contribution of -0.809704 . Thus, our single generalized coordinate shall be the first mode shape, depicted in Figure 3.

Table 1 summarizes the reduction in model complexity from the fully meshed model to the macro-model. Full 3D simulation of the capacitance of the system is performed 20 times, and this data is then fit to a [ $4 / 4$ ] rational polynomial as described above. The macromodels for each of the energy domains are then automatically exported to a SABER input file, and a black-box circuit element that represents this fixed-fixed beam structure is then simulated when driven by a variety of voltage profiles. Figure 4 depicts the response of our example device when actuated by a 100 volt saw wave with a rise time of $15 \mu \mathrm{s}$, a hold time of $10 \mu \mathrm{s}$, and a total period of $40 \mu \mathrm{s}$. Figure 5 depicts the response of our example device when actuated by an 80 volt saw wave with a rise time of $5 \mu \mathrm{s}$, a hold time of $10 \mu \mathrm{s}$, a fall time of $10 \mu \mathrm{s}$, and a total period of $40 \mu \mathrm{s}$.

This process has been applied to more complex structures,

$\begin{array}{rc}\text { \# nodes } \boldsymbol{N} & 534 \\ \text { \# modes } \boldsymbol{m} & 1 \\ \text { \# data points for } \boldsymbol{C}[\boldsymbol{q}] & 20 \\ \text { form of } \boldsymbol{C}(\boldsymbol{q}) & {[4 / 4]} \\ \text { \# terms } \boldsymbol{C}(\boldsymbol{q}) & 9 \\ \text { chi-squared } & 3.59 \mathrm{E}-24 \\ \text { measure of \%error } & 4.2 \mathrm{E}-04\end{array}$

Table 1. Summary of macromodel

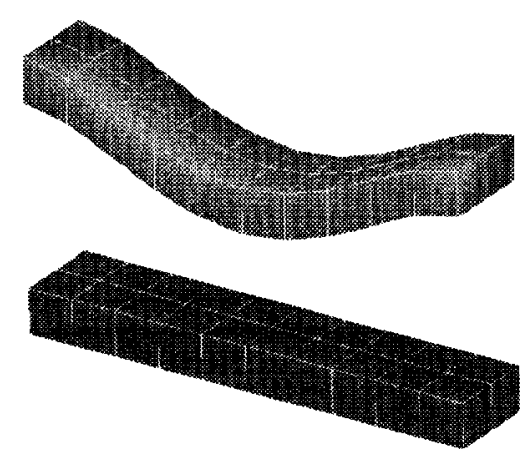

Figure 3. First mode shape for fixed-fixed beam example. Vertical scale expanded by factor of 20. This image connotes a negative value for the generalized coordinate, which is typical for this device under electrostatic actuation. 
such as a suspended plate supported by four beams of unequal thickness and actuated by an eccentric electrode. We have macromodeled this device using 5 mode shapes. A sample of simulation is given in Figure 6. We have also implemented this process to include non-linear strain-energy representations, but have discovered that large displacements result in excessively high strain energy calculations. This is because the system state determined from a superposition of node displacement vectors does not necessarily result in the minimum mechanical energy state of the system. This problem remains unsolved.

\section{CONCLUSIONS}

We have presented a method for macromodeling two conductor electromechanical devices without dissipation, and we have successfully applied this to the electrostatic actuation of a suspended beam and an elastically supported plate with an eccentric electrode and unequal springs. Furthermore, this technique is extendable to systems with other conservative energy domains.

An important benefit of this automated macromodeling approach is that it minimizes the number of iterative self-consistent coupled simulations that must be performed. A single such simulation is used to get estimates of the size of the modal workspace, but all simulations thereafter are single-energy-domain, hence fast. Further, the resulting dynamical nonlinear macromodel

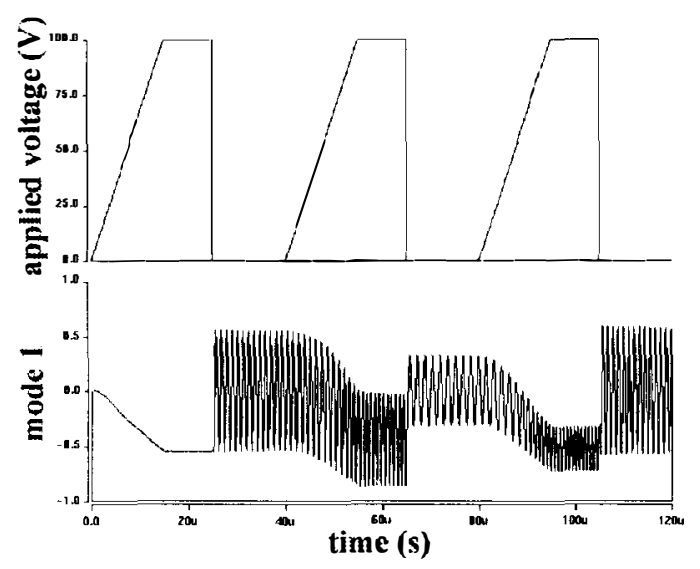

Figure 4. Response to a 100v saw wave with $15 \mu$ s rise, 10 $\mu$ s hold, and $40 \mu$ s period

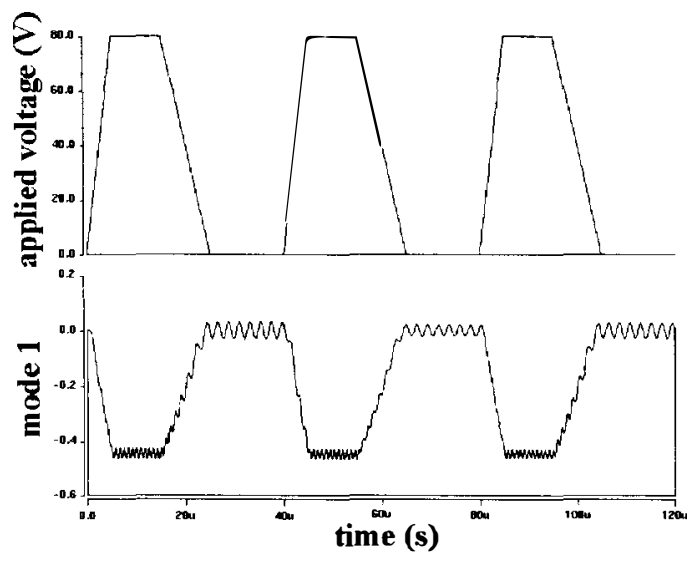

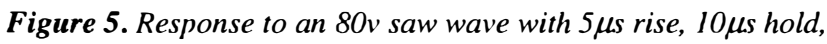
$10 \mu$ s fall, and $40 \mu$ s period is automatically exported to a SABER network element.

\section{ACKNOWLEDGMENTS}

This project was sponsored by the DARPA ETO MEMS program under contract J-FBI-95-215. The authors wish to thank Microcosm Technologies [9] for extensive cooperation in interfacing MEMCAD 3.2 with the macromodeling tools developed here.

\section{REFERENCES}

1. S. D. Senturia, "CAD for Microelectromechanical Systems", Proc. Transducers '95, Stockholm, Sweden; 25-29 June 1995, vol. 2, pp. 5-8.

2. S. D. Senturia, N. Aluru, and J. White, "Simulating the Behavior of MEMS Devices: Computational Methods and Needs", IEEE Computational Science and Engineering, vol. 4, no. 1, 1997, pp. 30-43.

3. H. A. Haus and J. R. Melcher, Electromagnetic Fields and Energy, Prentice Hall, 1989.

4. G. K. Ananthasuresh, R. K. Gupta, and S. D. Senturia, "An Approach to Macromodeling of MEMS for Nonlinear Dynamic Simulation", in Microelectromechanical Systems (MEMS), ASME Dynamic Systems \& Control (DSC) ser. Vol. 59, part of Proc. 1996 ASM Int'l Mechanical Engineering Congress and Exposition, Am. Soc. Of Mechanical Engineers, New York, 1996.

5. E. S. Hung, Y.-J. Yang, and S. D. Senturia, "Low-Order Models For Fast Dynamical Simulation of MEMS Microstructures," Transducers '97, pp. 1101-1104.

6. W. H. Press, S. A. Teukolsky, et al., Numerical Recipes in C: The Art of Scientific Computing, $2^{\text {nd }}$ edition, Cambridge University Press, 1995, pp. 681-688.

7. H. Goldstein, Classical Mechanics, $2^{\text {nd }}$ edition, AddisonWesley Publishing Company, 1981, pp. 16-21.

8. SABER, Analogy Inc., Beaverton, Oregon, http://www.analogy.com

9. Microcosm Technologies, Cambridge, Massachusetts, http://www.memcad.com

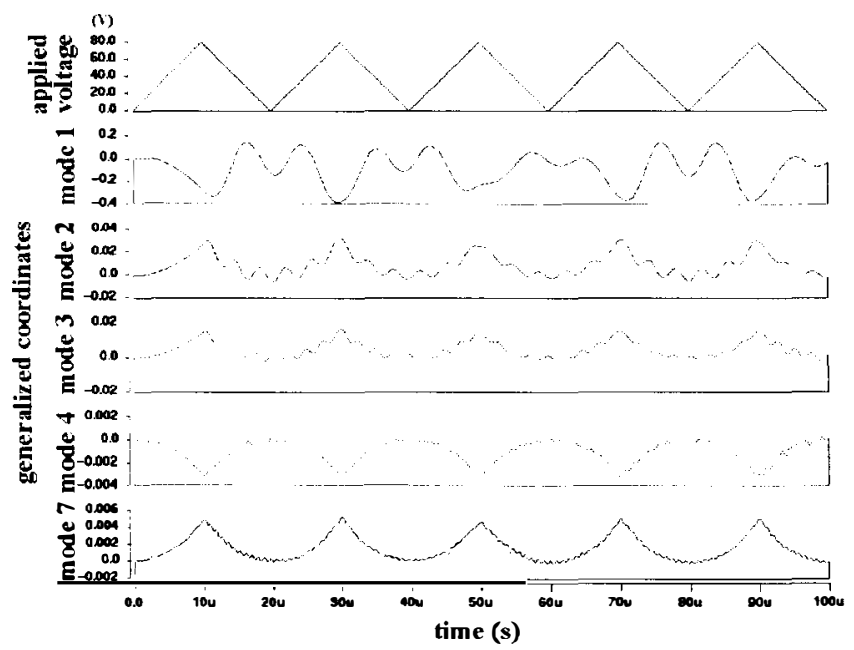

Figure 6. Response of asymmetrically supported plate 5-mode macromodel to an $80 \mathrm{v}$ saw wave with $20 \mu$ s period 